S.D. Ryder, D.J. Pisano, M.A. Walker, and K.C. Freeman, eds.

\title{
Gravitational Lens Statistics as a Probe of Halo Profiles
}

\author{
Masamune Oguri \\ Department of Physics, School of Science, University of Tokyo, Tokyo \\ 113-0033, Japan
}

\begin{abstract}
Recent development of the structure formation theory based on the cold dark matter scenario implies that a number of larger separation lensed quasars, for which a confirmed detection has not yet been achieved, will be observed in the ongoing large-scale surveys such as the $2 \mathrm{dF}$ survey and SDSS. We show that statistics of such large separation lenses can be a powerful probe of the density profile of dark halos. After we summarize the current status of the lens surveys in the 2dF and SDSS, we focus our discussion on what information can be extracted from these lens surveys. In addition, we also propose statistics of differential time delays between multiple images as an alternative probe of the density profile of dark halos.
\end{abstract}

\section{Introduction}

Statistics of lensed quasars offer invaluable information on the structure formation in the universe. In particular, larger separation $\left(\theta>6^{\prime \prime}\right)$ lensed quasars are key phenomena to understand "dark" side of our universe. The Cold Dark Matter (CDM) scenario, which is one of the most basic assumption in current standard structure formation model, predicts sufficiently cuspy mass distribution of dark matter halos. Since the image separation is a rough estimate of the mass of lensing objects, large separation lenses are expected to be produced by cluster-scale dark halos. In such massive dark halos, effect of baryon cooling is negligible, indicating that we can directly probe the mass distribution of dark matter. Therefore statistics of large separation lenses offer a useful test of the CDM paradigm (e.g., Oguri et al. 2002). Although several lens surveys have tried to find large separation lenses, they failed because lensing probability is much smaller than that of small separation lenses. For instance, a radio lens survey named CLASS (Cosmic Lens All-Sky Survey) searched for lenses with image separation $6^{\prime \prime}<\theta<15^{\prime \prime}$ from $\sim 10000$ quasars, but no lens system is found (Phillips et al. 2001).

\section{Large Separation Lenses in the 2dF Quasar Survey?}

Recently Miller et al. (2004) reported that they found 6 possible lenses with image separation $\theta>30^{\prime \prime}$ in the $2 \mathrm{dF}$ sample comprising $\sim 20000$ quasars. However, it is still premature to conclude that they are true lens systems, because of the lack of high-resolution spectra as well as deep-imaging of the systems. 
Indeed, we showed that the theoretically expected number of lenses with such large image separation $\left(\theta>30^{\prime \prime}\right)$ in the $2 \mathrm{dF}$ survey is much less than unity (Oguri 2003). But if this surprising result of the $2 \mathrm{dF}$ survey is true, then it becomes one of the most important problems (or "crises") in our understanding of structure formation theory based on the CDM scenario.

\section{Discovery of a Large Separation Lens from the SDSS}

In order to find unambiguous large separation lenses for the first time, we use the Sloan Digital Sky Survey (SDSS). The SDSS is still ongoing, but even currently it comprises $\sim 40000$ spectroscopically classified quasars, and is superior to the other surveys in several ways. We have searched for lens candidates in the SDSS data by selecting objects with similar colours; follow-up observations are needed to check whether the candidates are real lenses or not. Using this method, we have found a quadruple large separation lens system with a maximum image separation of 14.6 arcseconds (Inada et al. 2003; Oguri et al. 2003). The spectra of four quasar components were taken with LRIS on the Keck telescope, and turned out to be almost the same; all four components have typical quasar spectra with the same redshift $z=1.734$. Deep imaging observations by the Subaru Suprime-cam have unveiled the existence of a lensing cluster at $z=0.68$. We calculate the expected number of large separation lenses in the SDSS data. We constrain the SDSS finding in the $\alpha-\sigma_{8}$ plane, where $\alpha$ is the inner density profile of lens objects $\rho \propto r^{-\alpha}$ and $\sigma_{8}$ is the normalization of density fluctuations. Although the number depends on both $\alpha$ and $\sigma_{8}$, we find that the discovery in the SDSS and the lack of large separation lenses in the previous survey can be simultaneously explained with $\sigma_{8}=1.0_{-0.2}^{+0.4}$, if $\alpha=1.5$ which is the most plausible value in recent $N$-body simulations of the CDM model. Therefore the discovery offers strong support for the CDM scenario.

\section{References}

Inada, N., et al. 2003, Nature, submitted

Miller, L., Lopes, A. M., Smith, R. J., Croom, S. M., Boyle, B. J., Shanks, T., \& Outram, P. 2004, MNRAS, in press (astro-ph/0210644)

Oguri, M. 2003, MNRAS, 339, L23

Oguri, M., Taruya, A., Suto, Y., \& Turner, E. L. 2002, ApJ, 568, 488

Oguri, M., et al. 2003, ApJ, submitted

Phillips, P. M., et al. 2001, MNRAS, 328, 1001 